\title{
Surgical treatment of a 72-year-old patient with headache, hyponatremia and oculomotor nerve palsy: a case report and literature review
}

\author{
Hanchun Huang", Shenzhong Jiang", Chengxian Yang", Kan Deng, Renzhi Wang, Xinjie Bao \\ Department of Neurosurgery, Peking Union Medical College Hospital, Peking Union Medical College, Chinese Academy of Medical Sciences, \\ Beijing, China \\ \#These authors contributed equally to this work. \\ Correspondence to: Xinjie Bao. Department of Neurosurgery, Peking Union Medical College Hospital, Peking Union Medical College, Chinese \\ Academy of Medical Sciences, No. 1 Shuaifuyuan Hutong of Dongcheng District, Beijing 100730, China. Email: baoxinjie1@pumch.cn.
}

\begin{abstract}
Pituitary apoplexy is a life-threatening syndrome caused by acute infarction of the pituitary gland. The most common symptoms associated with pituitary apoplexy are headache, nausea, vomiting, visual symptoms, hypopituitarism, and altered mental status. Both oculomotor nerve palsy and hyponatremia are relatively rare complications of pituitary apoplexy. The treatment of pituitary apoplexy is controversial. We report a case of a 72-year-old man with severe headache, nausea, vomiting, confusion and left oculomotor nerve palsy, who was initially considered as posterior communicating artery aneurysm (PCOAA) based on the presenting symptoms. Initial biochemical evaluation showed severe hyponatremia, hormonal evaluation identified multiple pituitary hormone deficiency and enhanced magnetic resonance imaging showed a large pituitary adenoma with signs of hemorrhage. A diagnosis of pituitary apoplexy and secondary hypopituitarism was finally made. The patient was treated with intravenous hydrocortisone $100 \mathrm{mg}$ twice daily and oral levothyroxine $100 \mathrm{mg}$ once daily. Appropriate venous transfusion with sodium was also used concomitantly to correct hyponatremia. After seven days of treatment, the patient's serum electrolytes normalized and he gradually recovered alertness. Then, the patient underwent transsphenoidal surgery for tumor removal. Left ptosis and oculomotor nerve palsy completely recovered three months after surgery. Postoperatively, hormone replacement therapy was essential for the patient with $20 \mathrm{mg}$ hydrocortisone and $50 \mathrm{mg}$ levothyroxine once daily. During the last follow up 4 years later, the patient was still on hormonal replacement and in good condition. So, for patients with pituitary apoplexy, we have shown that a semielective surgery after conservative treatment when the patient becomes clinically stable and hypopituitarism has been corrected is a good approach.
\end{abstract}

Keywords: Pituitary apoplexy; hormone replacement; surgery; case report

Submitted May 21, 2020. Accepted for publication Nov 13, 2020.

doi: 10.21037 /gs-20-517

View this article at: http://dx.doi.org/10.21037/gs-20-517

\section{Introduction}

Pituitary apoplexy is caused by intratumoral hemorrhage or hemorrhagic infarction in pituitary adenomas. It is assumed that apoplexy is the product of intrinsic features of pituitary adenomas, and the tumor is vulnerable to spontaneous infarction or to acute ischemia by any event that acutely alters the balance between tumor perfusion and tumor metabolism (1). Its clinical symptoms include sudden severe headache $(89.7 \%)$, visual disturbances $(47.1 \%)$, visual field defects $(34.1 \%)$, cranial nerves palsy (39\%), and impairment in pituitary function which are often caused by compression of the enlarged sellar tumor (2). Meningeal irritation sometimes occurs because of blood or chemicals released from the pituitary adenoma. Cerebral 


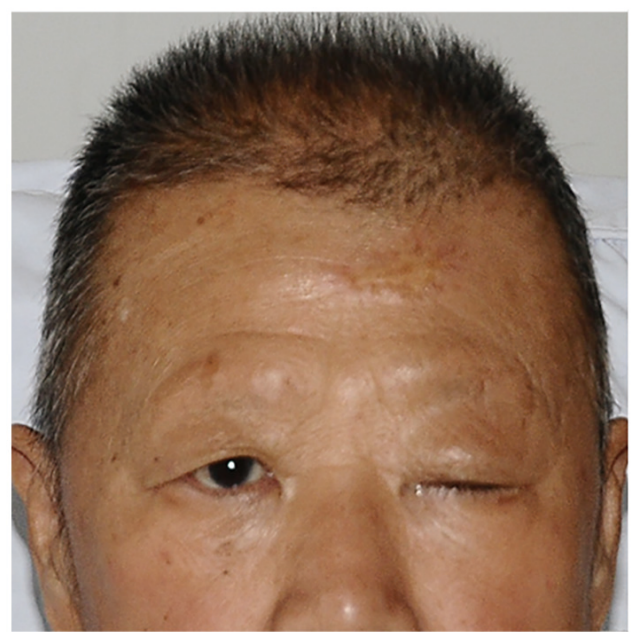

Figure 1 The patient cannot open his left eye, with complete ptosis and left eyeball abducent. We have got the patient's consent to publish his images.

infarct is a rare complication of pituitary apoplexy, and we have once reported a case with bilateral cerebral infarcts (3). In more than $80 \%$ of patients, apoplexy is the first presentation of the underlying pituitary tumor (4); symptoms vary and often overlap with other common diseases. Therefore, the diagnosis of pituitary apoplexy is challenging and its appropriate management still remains a matter of debate as no large-scale, randomized controlled studies comparing conservative with surgical management for pituitary apoplexy are available. Whether conservative treatment or surgical intervention is the most appropriate management remains a matter of debate (5). Five large retrospective studies comparing the outcomes of surgically and conservatively treated patients with pituitary apoplexy have shown that conservatively treated patients had higher rates of recovery from oculomotor palsies and visual deficits (6-10). Yet a recent systematic review shows the opposite result and it suggests surgical intervention for patients with visual defects and ocular palsy (11).

The optimal timing for surgery is a question as well if it is to be taken. Visual deficits used to be a neurosurgical emergency, but no difference in visual outcome is observed when surgery is performed within the first 3 days or during 4-7 days after the apoplectic event, whereas delays beyond one week may retard the return of visual function (12). Here, we present a case who underwent transsphenoidal surgery seven days after the pituitary apoplexy onset when he became clinically stable after conservative treatment. We present the following case in accordance with the CARE reporting checklist (available at http://dx.doi.org/10.21037/ gs-20-517).

\section{Case presentation}

On June 2015, a 72-year-old man presented to the emergency department of Peking Union Medical College Hospital with sudden severe headache, nausea, vomiting, confusion and left oculomotor nerve palsy. He has no brain injury and his wife denied the medical history of hypertension and brain surgery. No medical history of vascular risk factors was identified.

On examination, his Glasgow Coma Scale was 9/15, blood pressure was 115/65 $\mathrm{mmHg}$, heart rate was 67 beats per minute, the respiratory rate was 18 breaths per minute, and body temperature was $36.5^{\circ} \mathrm{C}$. The patient could open his right eye slightly in response to loud voice, with left eye complete ptosis and eyeball abducent (Figure 1). The diameter of left pupil was $5 \mathrm{~mm}$. Direct and indirect light reflection of left pupil was dull. Pathological reflexes were not induced and meningeal irritation sign was negative. In addition, the patient also suffered eyebrow fell off and hair loss, and his face was pale.

The first consideration may be subarachnoid hemorrhage caused by ruptured posterior communicating artery aneurysm (PCOAA). Therefore, a head computed tomography (CT) was performed to identify the subarachnoid hemorrhage. However, the head CT revealed no sign of subarachnoid hemorrhage, and a suspicious mass located in the sellar region (Figure 2). Because unruptured PCOAA can also lead to similar clinical presentations, we then performed brain magnetic resonance angiography (MRA) for this patient, which is noninvasive, more comfortable and safer compared with computed tomography angiography (CTA) and digital subtraction angiography (DSA). The result revealed no lesion of intracranial artery (Figure 3). Blood tests revealed remarkable hyponatremia (105 mmol/L).

This patient with new-onset severe hyponatremia and coexisted with neural symptoms and signs, neurologic diseases are still the most possible diagnosis. After exclusion of traumatic brain injury and subarachnoid hemorrhage caused by aneurysm, the suspicious mass in the sellar region in head CT brought to our attention. We suspected if there was lesion in the pituitary. Therefore, we performed an enhanced brain magnetic resonance image (MRI) focused on the sellar region, and hormones of the 


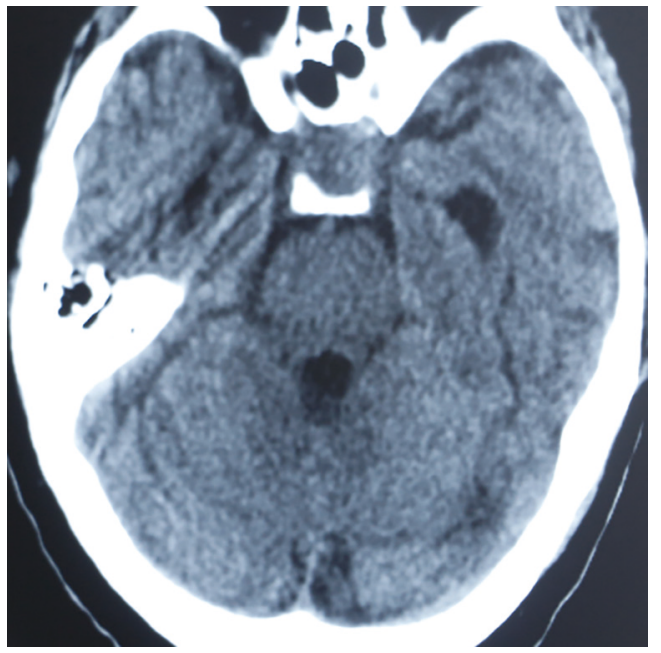

Figure 2 Head CT reveals no sign of subarachnoid hemorrhage, but a suspicious mass located in the sellar region. pituitary were detected. Tests of pituitary-related hormones indicated decreased levels of cortisol, thyroid hormone, and testosterone, while the growth hormone, insulinlike growth factor I, and prolactin were within normal ranges. Additionally, brain MRI revealed a lesion in the sellar region, with partially hyperintense on T1-weighted image and partially hypointense on T2-weighted image, indicative of hemorrhage in the lesion, which extended to the left cavernous sinus (Figure 4). Therefore, nonfunctional pituitary adenoma with pituitary apoplexy and hypopituitarism was considered.

The patient was then treated empirically with steroids, including intravenous hydrocortisone $100 \mathrm{mg}$ twice daily and oral levothyroxine $100 \mathrm{mg}$ once daily. Appropriate venous transfusion with sodium was used to correct hyponatremia. After seven days of treatment, the patient's
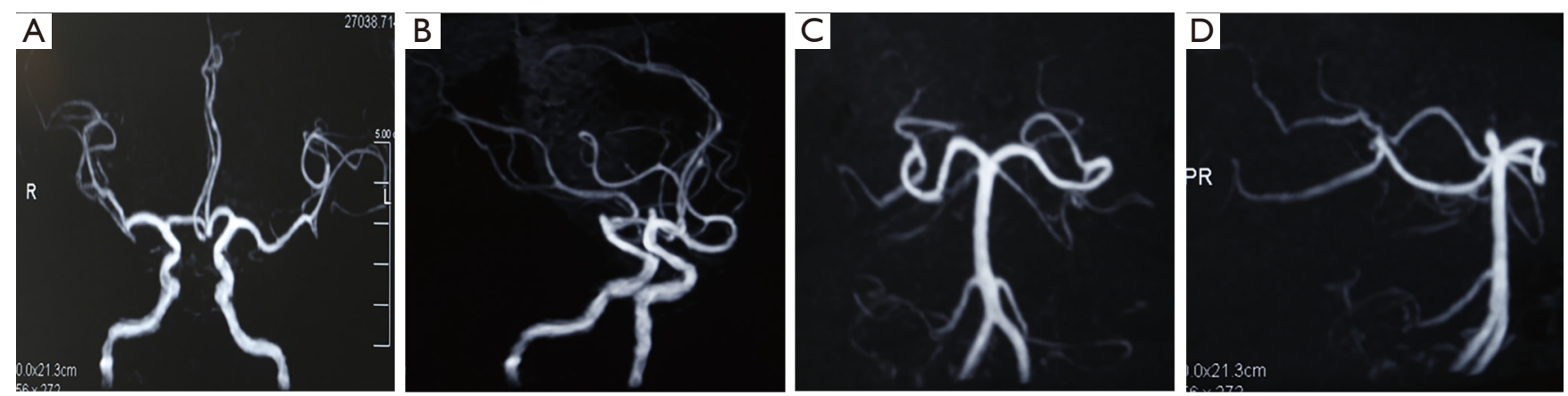

Figure 3 Brain magnetic resonance angiography reveals no aneurysm or other cerebrovascular lesion. Anteroposterior position (A) and lateral position (B) of front cerebral circulatory system; anteroposterior position (C) and lateral position (D) of back cerebral circulatory system.
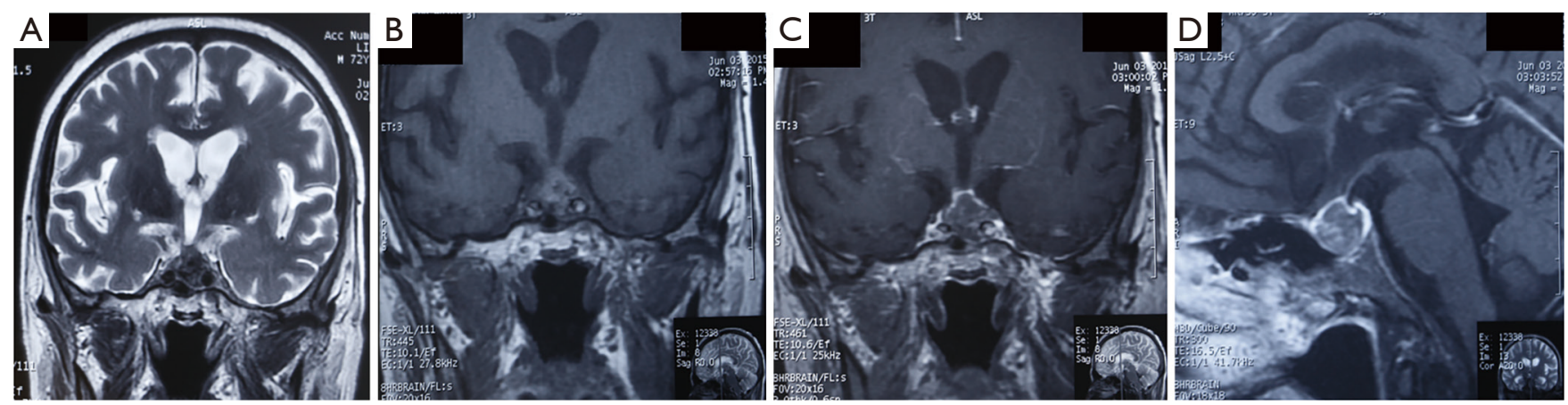

Figure 4 Magnetic resonance image reveals a lesion in the sellar region. It was partially hypointense on T2-weighted image (A) and partially hyperintense on T1-weighted image (B), which was indicative of intratumoral hemorrhage, with enhancement of the peritumoral surface (C,D). The pituitary adenoma extended to the left cavernous sinus causing compression of the oculomotor nerve (C). 

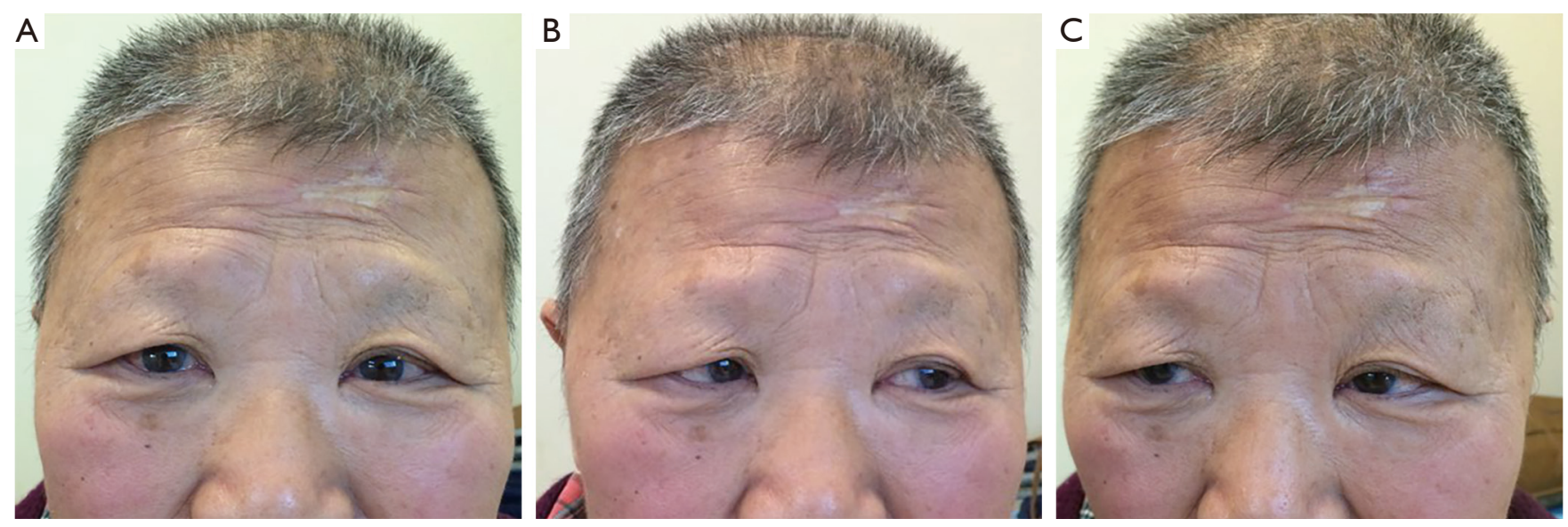

Figure 5 The manifestations of left oculomotor nerve palsy all disappeared about three months after surgery (A,B,C). We have got the patient's consent to publish his images.
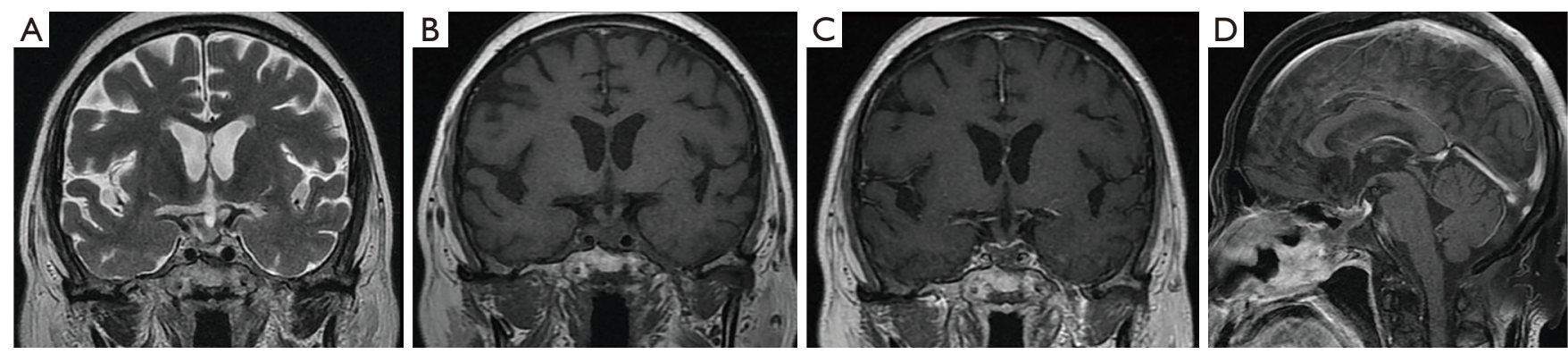

Figure 6 An enhanced brain MRI was performed eight months after surgery (A,B,C,D).

serum electrolytes normalized and he gradually recovered alertness. Then, the patient underwent transsphenoidal surgery for tumor removal. Histologic examinations revealed diffuse necrosis and hemorrhage in the nonfunctional pituitary adenoma. Left ptosis and oculomotor nerve palsy completely recovered three months after surgery (Figure 5). Hormone replacement therapy was essential for the patient with $20 \mathrm{mg}$ hydrocortisone and $50 \mathrm{mg}$ levothyroxine once daily, respectively. An enhanced brain MRI was performed eight months after surgery (Figure 6). The patient has no complaint and his neurological examination is normal. During the last follow up four years later, the patient was still on hormonal replacement and in good condition with no adverse and unanticipated events ever reported. The patient was very grateful for the medical services provided by our hospital and the great efforts we put into curing his disease. A timeline succinctly showed the historical and current information of the case (Figure 7).
All procedures performed in studies involving human participants were in accordance with the ethical standards of the institutional and/or national research committee(s) and with the Helsinki Declaration (as revised in 2013). Written informed consent was obtained from the patient.

\section{Discussion}

Pituitary apoplexy and subarachnoid hemorrhage show similar symptoms, including sudden headache, neck stiffness, and change in consciousness. In addition, cerebral aneurysm and pituitary apoplexy are detected together in $7.4 \%$ of patients with pituitary tumor $(5,13)$. The differential diagnosis between pituitary apoplexy and PCOAA was initially difficult in this case. MRA, CTA, or DSA can provide a very important clue to the diagnosis of cerebral aneurysm.

Cranial nerve dysfunction is due to pituitary tumor generally involved the third, fourth, fifth and sixth cranial 


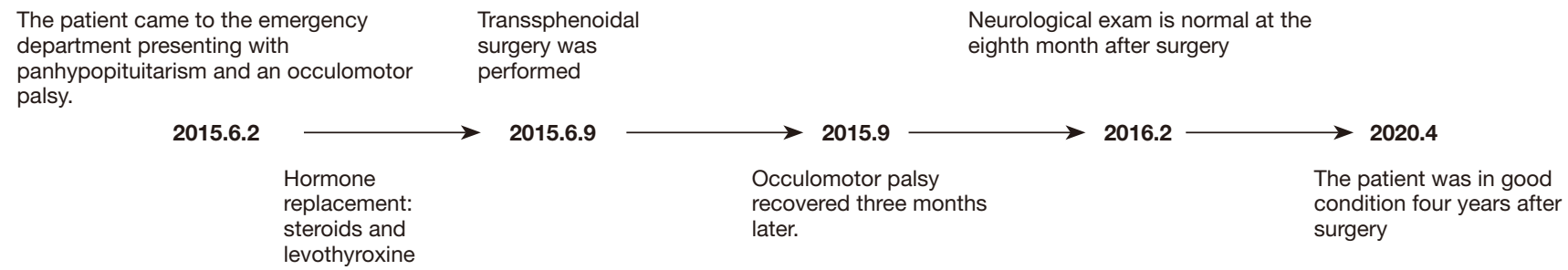

Figure 7 A timeline showing the historical and current information of the case.

nerves, which pass through the cavernous sinus (14). The third nerve (oculomotor nerve) is the most commonly involved cranial nerve. The causes of oculomotor nerve palsy include direct compression of the nerve by the tumor, transmission of the pressure on the cavernous sinus wall from tumor expansion, edematous expansion due to hemorrhage in the tumor, ischemic infarction of the tumor, and direct infiltration by the tumor (14). Those hypotheses are convictive, especially among the patients with cavernous sinus invasion. In this case, we did not find any obvious signs of cavernous sinus invasion during the operation. By reading the associated literatures, we found a new possible mechanism of oculomotor nerve palsy in pituitary apoplexy (15). Sudden increase of intratumoral pressure due to hemorrhage may result in compression of the oculomotor nerve at the entrance of the oculomotor trigone. With the erosion of the posterior clinoid process, tumor expansion can result in latero-posterior protrusion of the adenoma and compress the oculomotor trigone (15). Combined with the representation in brain MRI and findings during the operation, this mechanism is exactly reasonable for this patient.

Hyponatremia is a complicated electrolyte disturbance in clinical practice. Etiologies of hyponatremia are generally subdivided by a patient's volume status: hypovolemia, euvolemia or hypervolemia. Hypovolemic hyponatremia is a common etiology and responds well to volume repletion with normal saline. Euvolemic hyponatremia is mainly caused by the adrenal insufficiency, hypothyroidism, mineralocorticoid deficiency (primary adrenal insufficiency) or polydipsia (16).

Hyponatremia commonly occurs in patients suffering from traumatic brain injury, subarachnoid hemorrhage, or following intracranial procedures, with approximately $20 \%$ having a decreased serum sodium concentration to $<125 \mathrm{mmol} / \mathrm{L}$ (17). Severe hyponatremia was a presenting sign in $5.7 \%$ of patients with sellar lesions, most frequently in patients with arachnoid cysts, Rathke's cleft cysts, and pituitary apoplexy (18). Most of apoplectic patients coexist with secondary adrenal insufficiency, which is the main reason for hyponatremia. In secondary adrenal failure there is hypersecretion of antidiuretic hormone (ADH), mainly because cortisol deficiency results in increased hypothalamic secretion of Corticotropin-Releasing Hormone (CRH), an $\mathrm{ADH}$ secretagogue, as well as the decreased inhibition of $\mathrm{ADH}$ by cortisol (19). The increased $\mathrm{ADH}$ can lead to water retention and reduction in plasma sodium concentration. The other factors such as hypothyroidism and low plasma osmolality $\left(<275 \mathrm{mOsm} / \mathrm{kg} \mathrm{H}_{2} \mathrm{O}\right)$ also contribute to hyponatraemia (4). Pituitary apoplexy should be regarded as a differential diagnosis of hyponatremia.

For management, steroid therapy is mandatory in the first place as adrenal insufficiency is seen in $50-80 \%$ of patients with pituitary apoplexy onset and may be life threatening, potentially leading to severe hemodynamic problems and hyponatremia (5). However, whether conservative treatment or surgical intervention is the most appropriate management thereafter remains a matter of debate. Five large retrospective studies (6-10) have compared the outcomes of surgically and conservatively treated patients with pituitary apoplexy. Oculomotor palsies resolved completely in $75-100 \%$ of patients without surgery, versus in $31-57 \%$ of patients with surgery; visual acuity or visual filed defects were normalized in about $50-100 \%$ of conservatively treated cases, versus in $30-60 \%$ of patients in the surgically treated group; while the proportions of patients with post-treatment hypopituitarism were approximately the same in the surgical and conservative treatment groups. However, a recent systematic review shows the opposite result and it suggests surgical intervention for patients with visual defects and ocular palsy (11).

If the surgery is to be taken, then the optimal timing for it is a question as well. Emergent decompression surgery used to be considered necessary for pituitary apoplexy to save visual defects, but no difference in visual outcome is 
observed when surgery is performed within the first 3 days or during 4-7 days after the apoplectic event, whereas delays beyond 1 week may retard the return of visual function (12). In our case, transsphenoidal surgery was performed when the patient's general condition improved after seven days of steroid replacement therapy and the outcome was satisfactory.

The presenting case is challenging both diagnostically and therapeutically. For the diagnosis part, if the patient does not have a known history of pituitary tumor, MRA and MRI will be necessary for differentiation of vascular and pituitary diseases. DSA may not be the first choice in the emergency department as it is expensive and invasive, and complications may occur. For the treatment part, steroid replacement should be initiated as soon as the diagnosis is made. When patients' general condition improves after the replacement therapy, semi-elective transsphenoidal surgery is performed for tumor decompression. We do not recommend emergent surgery in patients who have remarkable adrenal insufficiency until the patient's hypopituitarism is corrected and general condition is stable. Because patients with unstable condition and pituitary dysfunction are at increased risk perioperatively and may not carry a good prognosis (12). Moreover, in this emergent setting, the surgery may be performed by the on-call neurosurgical team rather than by an experienced pituitary surgeon, which increases the risk of adverse events as well. Emergent neurosurgical decompression is necessary only when the patient has significant and deteriorating neuroophthalmic signs or declining level of consciousness in spite of the steroid replacement (20). In a word, early diagnosis and treatment of pituitary apoplexy are crucial for avoiding complications and preventing permanent visual defects

The strength of this case is its detailed information of the clinical findings, diagnostic assessments and therapeutic interventions. It has its limitation. As the treatment of pituitary apoplexy is controversial, the experience of one special case may not apply to all patients, thus further prospective studies are required.

In conclusion, patients with severe hyponatremia and no obvious cause should undergo pituitary hormonal evaluation to identify the function of pituitary. The treatment of pituitary apoplexy is controversial. However, both the hormone replacement therapy and other supporting administration are regarded as essential treatments. Transsphenoidal surgery is the first choice for surgical decompression to save visual acuity. In our experience, transsphenoidal surgery should be performed when the patient's general condition improved after a short term of hormone replacement therapy, and the prognosis will be better.

\section{Acknowledgments}

We express many thanks to the patient for generously authorizing us to share his rare case.

Funding: None.

\section{Footnote}

Reporting Checklist: The authors have completed the CARE reporting checklist. Available at http://dx.doi.org/10.21037/ gs-20-517

Peer Review File: Available at http://dx.doi.org/10.21037/gs20-517

Conflicts of Interest: All authors have completed the ICMJE uniform disclosure form (available at http://dx.doi. org/10.21037/gs-20-517). The authors have no conflicts of interest to declare.

Ethical Statement: The authors are accountable for all aspects of the work in ensuring that questions related to the accuracy or integrity of any part of the work are appropriately investigated and resolved. All procedures performed in studies involving human participants were in accordance with the ethical standards of the institutional and/or national research committee(s) and with the Helsinki Declaration (as revised in 2013). Written informed consent was obtained from the patient.

Open Access Statement: This is an Open Access article distributed in accordance with the Creative Commons Attribution-NonCommercial-NoDerivs 4.0 International License (CC BY-NC-ND 4.0), which permits the noncommercial replication and distribution of the article with the strict proviso that no changes or edits are made and the original work is properly cited (including links to both the formal publication through the relevant DOI and the license). See: https://creativecommons.org/licenses/by-nc-nd/4.0/.

\section{References}

1. Oldfield EH, Merrill MJ. Apoplexy of pituitary adenomas: the perfect storm. J Neurosurg 2015;122:1444-9. 
2. Singh TD, Valizadeh N, Meyer FB, et al. Management and outcomes of pituitary apoplexy. J Neurosurg 2015;122:1450-7.

3. Han F, Peng B, Gao S, et al. Clinical Reasoning: a 42-yearold man with severe headache, fever, and acute coma. Neurology 2014;82:e9-13.

4. Fountas A, Andrikoula M, Tsatsoulis A. A 45 year old patient with headache, fever, and hyponatraemia. BMJ 2015;350:h962.

5. Briet C, Salenave S, Bonneville JF, et al. Pituitary Apoplexy. Endocr Rev 2015;36:622-645.

6. Ayuk J, McGregor EJ, Mitchell RD, et al. Acute management of pituitary apoplexy--surgery or conservative management? Clin Endocrinol (Oxf) 2004;61:747-52.

7. Bujawansa S, Thondam SK, Steele C, et al. Presentation, management and outcomes in acute pituitary apoplexy: a large single-centre experience from the United Kingdom. Clin Endocrinol (Oxf) 2014;80:419-24.

8. Gruber A, Clayton J, Kumar S, et al. Pituitary apoplexy: retrospective review of 30 patients--is surgical intervention always necessary? Br J Neurosurg 2006;20:379-85.

9. Leyer C, Castinetti F, Morange I, et al. A conservative management is preferable in milder forms of pituitary tumor apoplexy. J Endocrinol Invest 2011;34:502-9.

10. Sibal L, Ball SG, Connolly V, et al. Pituitary apoplexy: a review of clinical presentation, management and outcome in 45 cases. Pituitary 2004;7:157-63.

11. Tu M, Lu Q, Zhu P, et al. Surgical versus non-surgical treatment for pituitary apoplexy: A systematic review and meta-analysis. J Neurol Sci 2016;370:258-262.

Cite this article as: Huang $\mathrm{H}$, Jiang S, Yang C, Deng K, Wang R, Bao X. Surgical treatment of a 72-year-old patient with headache, hyponatremia and ocu-lomotor nerve palsy: a case report and literature review. Gland Surg 2021;10(1):364-370. doi: $10.21037 / g s-20-517$
12. Turgut M, Ozsunar Y, Basak S, et al. Pituitary apoplexy: an overview of 186 cases published during the last century. Acta Neurochir (Wien) 2010;152:749-61.

13. Lee KC, Lee KS, Shin YS, et al. Surgery for posterior communicating artery aneurysms. Surg Neurol 2003;59:107-13.

14. Kim SH, Lee KC, Kim SH. Cranial nerve palsies accompanying pituitary tumour. J Clin Neurosci 2007;14:1158-62.

15. Kobayashi H, Kawabori M, Terasaka S, et al. A possible mechanism of isolated oculomotor nerve palsy by apoplexy of pituitary adenoma without cavernous sinus invasion: a report of two cases. Acta Neurochir (Wien) 2011;153:2453-6; discussion 2456.

16. Sahay M, Sahay R. Hyponatremia: A practical approach. Indian J Endocrinol Metab 2014;18:760-71.

17. Kleindienst A, Hannon MJ, Buchfelder M, et al. Hyponatremia in Neurotrauma: The Role of Vasopressin. J Neurotrauma 2016;33:615-24.

18. Bordo G, Kelly K, McLaughlin N, et al. Sellar Masses that Present with Severe Hyponatremia. Endocr Pract 2014;20:1178-86.

19. Ma XM, Aguilera G. Differential regulation of corticotropin-releasing hormone and vasopressin transcription by glucocorticoids. Endocrinology 1999;140:5642-50.

20. Rajasekaran S, Vanderpump M, Baldeweg S, et al. UK guidelines for the management of pituitary apoplexy. Clin Endocrinol (Oxf) 2011;74:9-20. 